\title{
Impacto dos tratamentos de fissuras labiopalatinas na qualidade de vida: avaliação pelo CLEFT-Q
}

\author{
Impact of cleft lip and palate treatments on quality of life: CLEFT-Q assessment \\ Impacto de los tratamientos para el labio leporino y el paladar hendido en la calidad de vida: \\ Evaluación CLEFT-Q
}

Recebido: 28/09/2021 | Revisado: 09/10/2021 | Aceito: 12/10/2021 | Publicado: 13/10/2021

\author{
Maria Sueli Marques Soares \\ ORCID: https://orcid.org/0000-0003-0712-8456 \\ Universidade Federal da Paraíba, Brasil \\ E-mail: msuelimarques@gmail.com \\ Mariana Silva Barros \\ ORCID: https://orcid.org/0000-0001-9298-7196 \\ Universidade Federal de Sergipe, Brasil \\ E-mail: marianab.odonto@gmail.com \\ Ana Carolina Lyra de Albuquerque \\ ORCID: https://orcid.org/0000-0002-6532-5020 \\ Universidade Federal da Paraíba, Brasil \\ E-mail: lina.lyra@gmail.com \\ Rosa Helena Wanderley Lacerda \\ ORCID: https://orcid.org/0000-0003-0011-6487 \\ Universidade Federal da Paraíba, Brasil \\ E-mail: rhelenawanderley@msn.com \\ Alexandre Rezende Vieira \\ ORCID: https://orcid.org/0000-0003-3392-6881 \\ Universidade de Pittsburg, Estados Unidos da América \\ E-mail: alexandre_vieira@pitt.edu
}

\begin{abstract}
Resumo
Introdução: A perspectiva do paciente com fissura labiopalatina (FLP) é importante para avaliar resultados obtidos nos tratamentos, podendo contribuir para melhor qualidade de vida. Objetivos: Através do CLEFT-Q, avaliar a influência do tratamento na qualidade de vida do paciente. Métodos: Estudo transversal observacional, com 100 indivíduos com FLP, os quais responderam o CLEFT-Q. Resultados: A média de idade dos participantes foi $16 \pm 4,9$ anos. O tipo de FLP mais frequente foi o transforame, com $85 \%$. Os tratamentos realizados foram: queiloplastia, palatoplastia, enxerto ósseo, rinoplastia e tratamento ortodôntico. Os escores CLEFT-Q mais baixos foram nas Escalas de Aparência, relacionados à aparência do nariz e narinas e, os maiores foram nas Escalas de Qualidade de vida, relacionados a aspectos psicológico e escolar. Houve correlação estatisticamente significante entre os tratamentos e pelo menos uma Escala nos domínios Aparência, Qualidade de vida e Função facial, exceto para rinoplastia. O tratamento ortodôntico teve correlação negativa com todos os domínios, com associação estatisticamente significante nas Escalas face, narinas, dentes, lábios, dificuldade da fala, escolar, social e fala, exceto para nariz, psicológico e comer/beber. A escala fala teve correlação estatisticamente significante com palatoplastia, enxerto ósseo e tratamento ortodôntico e, a Escala comer/beber teve com enxerto ósseo. Conclusões: Pacientes com FLP sentem insatisfação com sua aparência facial, especialmente, quanto ao nariz, narinas e dentes. Os tratamentos impactaram na percepção do paciente quanto a aparência, qualidade de vida relacionada à saúde e função facial. A queiloplastia e o enxerto ósseo impactaram positivamente na qualidade de vida do paciente com FLP.
\end{abstract}

Palavras-chave: Fenda labial; Fissura palatina; Qualidade de vida.

\begin{abstract}
Introduction: The perspective for patients with cleft lip and/or palate (CLP) is important in assessing treatment outcomes and contributing to a better quality of life. Objectives: To evaluate the influence of treatment patients' quality of life with CLP using CLEFT-Q. Methods: Observational cross-sectional study, with 100 individuals attended in a CLP Service. Results: The mean age of the sample was $16 \pm 4.9$ years. The most frequent cleft type was cleft lip/palate and the most prevalent, transforamen (85\%). The treatments performed were: cheiloplasty, palatoplasty, bone grafting, rhinoplasty and orthodontic treatment. The lowest Cleft-q scores were in Appearance and related to appearance of nose and nostrils while the highest were observed in Quality of life Scales as psychological and school. There was a statistically significant correlation between treatments performed and at least one Scale of Appearance,
\end{abstract}


Quality of Life and Facial Function, except for rhinoplasty. Orthodontic treatment showed a negative correlation with the three domains, and the association was statistically significant in Face, Nostrils, Teeth, Lips, Difficulty Speaking, School, Social and Speech Scales, except for Nostrils, Psychological and Eating/Drinking. The speech scale showed statistically significant correlation with palatoplasty, bone grafting and orthodontic treatment and eating/drinking scale showed statistically significant correlation only with bone grafting. Conclusions: The results suggests that the patients studied were dissatisfied with their facial appearance, especially regarding nose, nostrils and teeth. All treatments impacted the patients' perception of their appearance, health-related quality of life and facial function. Cheiloplasty and bone grafting had a positive impact on patients' quality of life.

Keywords: Cleft lip; Cleft palate; Quality of life.

\section{Resumen}

Introducción: La perspectiva del paciente con fisura labiopalatina (FLP) es importante en la evaluación de los resultados del tratamiento y puede contribuir a una mejor calidad de vida. Objetivos: Utilizar el CLEFT-Q para evaluar la influencia del tratamiento en la calidad de vida de pacientes con FLP. Métodos: Estudio observacional transversal con 100 individuos atendidos en un Servicio de FLP y que responderan el CLEFT-Q. Resultados: La edad media de los participantes fue 16 $\pm 4,9$ años. El tipo de hendidura más frecuente fue la de labio/paladar hendido, siendo el transforamen el más prevalente $(85 \%)$. Los tratamientos realizados fueron: queiloplastia, palatoplastia, injerto óseo, rinoplastia y tratamiento de ortodoncia. Las puntuaciones más bajas del CLEFT-Q estaban relacionadas con la Apariencia, principalmente de la nariz y las fosas nasales, y las más altas eran las psicológicas y escolares para la Calidad de vida. Hubo una correlación estadísticamente significativa entre los tratamientos realizados y al menos una escala de los dominios Apariencia, Calidad de vida y Función facial, excepto para la rinoplastia. El tratamiento de ortodoncia mostró una correlación negativa con los tres dominios, y la asociación fue estadísticamente significativa para la cara, las fosas nasales, los dientes, los labios, la dificultad para hablar, la escuela, lo social y el habla, excepto para la nariz, lo psicológico y comer/beber. El habla mostró una correlación estadísticamente significativa con la palatoplastia, el injerto óseo y el tratamiento de ortodoncia, y el comer/beber una correlación estadísticamente significativa con el injerto óseo. Conclusiones: Pacientes com FLP sentían insatisfacción por el aspecto facial, especialmente en lo que respecta a la nariz, las fosas nasales y los dientes. Los tratamientos repercutieron en la percepción de la apariencia del paciente, la calidad de vida relacionada con la salud y la función facial. La queiloplastia y el injerto óseo tuvieron un impacto positivo en la calidad de vida de pacientes con FPL.

Palabras clave: Labio leporino; Paladar hendido; Calidad de vida.

\section{Introdução}

Problemas de saúde bucal podem influenciar na Qualidade de Vida (QV) das pessoas devido à dor, desconforto, limitações e problemas estéticos, afetando a vida social, a alimentação, as atividades diárias e o bem-estar do indivíduo. Avaliações relatadas pelo paciente são, particularmente importantes ao examinar condições crônicas com trajetória de tratamentos prolongados, como no caso de fenda labial e palatina (Sischo et al., 2017).

Fissura labiopalatina (FLP) é um dos tipos mais comuns de lesões maxilofaciais congênitas (Sato et al., 2021; Ono et al., 2015) com incidência global de 1 para 700 nascidos vivos (Raghavan et al., 2018) e, no Brasil atinge 1 em cada 650 nascidos vivos (Ministério da Saúde, Brasil).

Devido às necessidades especiais associadas à fissura, incluindo desafios de fala/ linguagem, diferenças faciais, desenvolvimento dentário atípico, má oclusão, dificuldades de aprendizagem, infecções de ouvido crônicas e sequelas psicossociais associadas, o atendimento ao paciente com FLP é realizado em equipe multidisciplinar, envolvendo cirurgiões plásticos, dentistas, psicólogos, assistentes sociais e fonoaudiólogos (Lewis et al., 2017). Estes profissionais fornecem avaliações e intervenções contínuas desde a infância até o final da adolescência ou idade adulta jovem. Sischo et al. (2017) presumem que as operações sequenciais melhoram o bem-estar do paciente, embora haja poucas evidências empíricas disponíveis para apoiar essa afirmação.

Três procedimentos principais são instituídos para correção da deformidade, a queiloplastia, a palatoplastia e o enxerto ósseo alveolar. Comumente poderão ser adicionados procedimentos secundários para melhorar a função e estética. Dentre estes, a correção labial de um resultado insatisfatório, correção da disfunção velofaríngea, após palatoplastia; cirurgia ortognática para correção maxilar; rinoplastia para deformidade nasal secundária e cirurgia para incompetência velofaríngea 
(Raghavan et al., 2018). Geralmente, esperam-se resultados mais desejáveis quando são aplicados protocolos que preconizam que as estruturas de tecidos moles, como o lábio e a membrana mucosa devem ser tratadas cirurgicamente em um período relativamente curto desde o nascimento, até 3 meses. Para a palatoplastia dura com enxerto ósseo, se preconiza realizar o procedimento entre 8 e 10 anos de idade (Kuwada et al., 2021).

A autopercepção pode ter impacto significativo no estado psicológico e no status social humano, afetando a autoestima e a qualidade de vida (QV) geral do indivíduo. Existem fatores que desempenham importante papel na satisfação do paciente que são impossíveis se objetivar em alguns métodos de avaliação, sendo necessário valorizar uma comunicação clara entre o profissional de saúde e o paciente com FLP, especialmente em relação às suas expectativas, percepção e satisfação com os resultados de cirurgias (Mulder et al., 2019; Stiernman et al., 2021). Assim, considerar a perspectiva do paciente em relação à saúde oral e qualidade de vida relacionada à saúde é importante porque, no decorrer do longo período de tratamentos, ocorrem transformações anatômicas, estéticas, fonéticas e psicológicas e os resultados das intervenções cirúrgicas e demais tratamentos, nem sempre correspondem às expectativas do paciente, podendo levar ao desequilíbrio emocional e à dificuldade de convívio social e interferir negativamente na qualidade de vida. (Sischo et al., 2017; Moi et al., 2020; Lewis et al., 2017).

Investigar a perspectiva do paciente por meio de instrumento de autoavaliação pode aumentar a compreensão dos profissionais sobre as preocupações desse indivíduo com sua saúde (Stiernman et al., 2021), o que permite um melhor entendimento das necessidades do paciente ou aponta a necessidade de ajuste do tipo de tratamento para melhor adequação de sua condição psicossocial. O CLEFT-Q é um método avaliativo que analisa objetivamente a percepção do indivíduo quanto à autoimagem, visando representar de forma mais próxima, à realidade de como indivíduos com FLP se sentem. Os resultados obtidos podem impactar nos protocolos de tratamento. (Riff et al., 2019).

$\mathrm{Na}$ literatura ainda são escassos estudos neste campo e tem-se observado resultados controversos ou difíceis de serem analisados devido a diferenças metodológicas, havendo necessidade de estudos que utilizem ferramentas que possam ser aplicadas à rotina de atendimento odontológico e avaliem os efeitos psicossociais dos tratamentos em pacientes com FLP. Diante do exposto, o objetivo do presente estudo foi avaliar a possível correlação entre alguns tipos de tratamentos e tipos de FLP e os resultados do índice CLEFT-Q.

\section{Metodologia}

Foi realizado um estudo transversal, observacional, com uma amostra de conveniência (Estrela, 2018), composta por 100 pacientes com fissura labiopalatina (FLP), de ambos os sexos, registrados no Serviço de Fissuras Labiopalatinas em um Hospital Universitário e, atendidos entre os meses de julho e agosto de 2019. Os critérios de inclusão foram: indivíduos com idade de 8 e 29 anos, possuir fissura de lábio e/ou palato e, ser funcionalmente independente. Aqueles com FLP associada à síndrome e com comprometimento da capacidade intelectual ou cognitiva foram excluídos. A partir do prontuário dos pacientes, foram coletados dados sobre idade, sexo, tipo de fissura e os tipos de tratamentos aos quais os pacientes haviam sido submetidos. Tais tratamentos incluíram queiloplastia, palatoplastia, enxerto ósseo, rinoplastia e tratamento ortodôntico, sendo registrada a idade em que o paciente realizou cada tratamento.

O índice CLEFT-Q representa um instrumento de autorrelato, rigorosamente desenvolvido para avaliar pacientes com FLP e, com idade entre 8 e 29 anos (Klassen et al., 2018). Para utilizá-lo no presente estudo foi obtida autorização da McMaster Industry Liaison Office (MILO).

Durante uma consulta odontológica os pacientes foram selecionados e responderam o instrumento CLEFT-Q validado em português brasileiro (Klassen et al., 2018) e impresso em papel. O instrumento é composto por 13 Escalas para avaliar três domínios: Aparência (face, nariz, narinas, dentes, lábios, cicatriz da fissura labial e mandíbula/maxila), Qualidade de Vida 
(função psicológica, função escolar, função social e dificuldade da fala) e Função Facial (Fala; Comer/beber), (Tsangaris et al. 2017). Quanto à Aparência se avalia a percepção do paciente sobre sua face, seu nariz e narinas, dentes, lábios e possíveis cicatrizes, quando sorri, conversa ou aparece em fotografias. No domínio Qualidade de Vida é avaliado o estado psicológico do paciente, sua vida escolar e social, considerando a participação, aceitação e segurança no convívio social, além da dificuldade de falar em público e ser compreendido ou o quanto se sente confiante em situações que exigem exposição (Tsangaris et al., 2017). No domínio Função Facial são avaliadas Escalas relacionadas à capacidade de falar e à alimentação para saber se o paciente evita alguns tipos de alimentos, se tenta consumi-los mais lentamente ou se sai líquido pelo nariz durante a alimentação, o que pode gerar constrangimento e reclusão social (Namankany et al., 2018). As Escalas deste instrumento são independentes e o paciente pode responder apenas as que ele considerar relevantes.

O valor de cada domínio é obtido pela soma dos valores das respostas de suas Escalas, podendo ser de 0 e 100 pontos e, os maiores escores representam melhores resultados. No presente estudo foram considerados satisfatórios resultados iguais ou superiores a 70 pontos e, quando menores foram considerados insatisfatórios, segundo Klassen et al. (2018). Quanto à escala comer e beber, a pontuação varia de 9 a 36 pontos e, as médias maiores representam mais problemas durante a alimentação (Tsangaris et al.,2017). No presente estudo foram considerados satisfatórios valores acima de 25 pontos e, valores menores considerados insatisfatórios (Klassen et al., 2018).

Os dados obtidos foram processados no SPSS (Statistical Package for Social Science, versão 23, IBM), sendo realizada análise descritiva e, como os dados gerados pelo CLEFT-Q são ordinais, foi usado teste não paramétrico, coeficiente de correlação de Spearman, considerando significantes valores de $\mathrm{P}>5 \%$.

O estudo seguiu as orientações do Comitê de Ética em Pesquisa do Centro de Ciências da Saúde da Universidade Federal da Paraíba, sob o número 15398919.0.0000.5188.

\section{Resultados e Discussão}

Na Tabela 1 observa-se que do total da amostra estudada 52\% dos participantes eram homens com idade variando de 8 a 29 anos e, média de idade de $16 \pm 4,9$ anos. O tipo de fissura mais frequente envolvia lábio e palato, sendo as fissuras transforame mais prevalentes, com $85 \%$ dos casos.

Também estão apresentados na Tabela 1, os tipos de tratamentos aos quais os pacientes da amostra foram submetidos, estando distribuídos por idade. Observa-se que os procedimentos de queiloplastia e tratamento ortodôntico foram os mais frequentes, enquanto que a rinoplastia o procedimento menos realizado. Até os 12 meses de idade $81 \%$ dos pacientes havia realizado queiloplastia e, $43 \%$ realizado palatoplastia. Enxerto ósseo foi realizado em maior percentual em adolescentes de 10 a 17 anos, enquanto que a rinoplastia entre aqueles com 13 e 18 anos de idade. 
Tabela 1. Distribuição da amostra.

\begin{tabular}{|c|c|c|c|c|c|c|c|}
\hline Sexo & Número & Percentual & & & & & \\
\hline Masculino & 52 & 52 & & & & & \\
\hline Feminino & 48 & 48 & & & & & \\
\hline $\begin{array}{l}\text { Média de idade / desvio } \\
\text { padrão }\end{array}$ & $16 \pm 4,9$ & & & & & & \\
\hline 8 a 12 anos & 24 & 24 & & & & & \\
\hline 13 a 17 anos & 41 & 41 & & & & & \\
\hline 18 a 29 anos & 35 & 35 & & & & & \\
\hline \multicolumn{8}{|l|}{ Tipo de Fissura } \\
\hline Transforame unilateral & 51 & 51 & & & & & \\
\hline Transforame bilateral & 34 & 34 & & & & & \\
\hline Pré-forame unilateral & 8 & 8 & & & & & \\
\hline Pré-forame bilateral & 1 & 1 & & & & & \\
\hline Pós-forame & 6 & 6 & & & & & \\
\hline Tipo de tratamento & & $\begin{array}{l}\text { até } 12 \\
\text { meses }\end{array}$ & 2 a 5 anos & 6 a 9 anos & $\begin{array}{l}10-13 \\
\text { anos }\end{array}$ & $\begin{array}{l}14 \text { a } 17 \\
\text { anos }\end{array}$ & $\begin{array}{l}18 \text { a } 29 \\
\text { anos }\end{array}$ \\
\hline Queiloplastia & 93 & 81 & 10 & 1 & 1 & 0 & 0 \\
\hline Palatoplastia & 85 & 43 & 28 & 7 & 5 & 1 & 1 \\
\hline Enxerto ósseo & 46 & 0 & 1 & 7 & 18 & 14 & 6 \\
\hline Rinoplastia & 17 & 4 & 0 & 0 & 10 & 0 & 3 \\
\hline Ortodôntico & 93 & 0 & 0 & 26 & 39 & 12 & 16 \\
\hline
\end{tabular}

Fonte: Dados da pesquisa (2019).

As pontuações médias das Escalas do CLEFT-Q obtidas no presente estudo, estão demostradas na tabela 2. Observase que os escores mais baixos estão nas Escalas do domínio Aparência e relacionados à aparência do nariz e das narinas. Enquanto que os maiores escores foram observados nas Escalas relacionadas ao domínio Qualidade de vida, particularmente nas Escalas psicológico e escolar. No domínio Função facial, a Escala fala teve baixo escore. 
Tabela 2. Valores CLEFT-Q na amostra estudada, por Domínios e Escalas.

\begin{tabular}{lcccc}
\hline CLEFT-Q & (n) & mínimo & máximo & média \pm desvio padrão \\
\hline Aparência & & & & \\
Face & 97 & 20 & 100 & $58,5 \pm 15$ \\
Nariz & 91 & 0 & 100 & $49,7 \pm 17$ \\
Narinas & 92 & 0 & 100 & $49,7 \pm 24$ \\
Dentes & 97 & 9 & 100 & $52,7 \pm 17$ \\
Lábios & 95 & 9 & 100 & $57,5 \pm 18$ \\
Cicatriz labial & 95 & 0 & 100 & $59,3 \pm 26$ \\
Maxilares & 96 & 0 & 100 & $61,5 \pm 24$ \\
Qualidade de vida relacionada à & & & & \\
Saúde & & & & \\
Dificuldade de fala & 95 & 12 & 100 & $67,5 \pm 22$ \\
Psicológico & 100 & 15 & 100 & $72,9 \pm 20$ \\
Escolar & 98 & 27 & 100 & $70,3 \pm 17$ \\
Social & 98 & 25 & 100 & $67,7 \pm 16$ \\
Função facial & & & & \\
Fala & 99 & 11 & 36 & $58,2 \pm 21$ \\
Comer/beber & & & & \\
\hline & & & & \\
(n) & 97 & & & \\
\end{tabular}

\footnotetext{
n (número de pacientes).

(n) número de casos

Fonte: Dados da pesquisa (2019)
}

Foi observada correlação estatisticamente significante entre os tratamentos realizados em pelo menos uma Escala dos domínios Aparência, qualidade de vida e função facial, exceto para rinoplastia, conforme apresentado na tabela 3 . Observa-se também que o tratamento ortodôntico apresentou correlação negativa com todos os três domínios (Aparência, qualidade de vida e função facial), sendo a associação estatisticamente significante nas Escalas face, narinas, dentes, lábios, dificuldade de falar, escolar, social e fala, exceto nas Escalas nariz, psicológico e comer/beber. 
Tabela 3. Correlação entre CLEFT-Q e tipos de tratamentos na amostra estudada.

\begin{tabular}{lccccc}
\hline & \multicolumn{3}{c}{ Tratamentos } \\
$\begin{array}{l}\text { Cleft-Q } \\
(\mathrm{n}=93)\end{array}$ & $\begin{array}{c}\text { Queiloplastia } \\
(\mathrm{n}=\mathbf{8 5})\end{array}$ & $\begin{array}{c}\text { Palatoplastia } \\
(\mathrm{n}=46)\end{array}$ & $\begin{array}{c}\text { Enxerto ósseo } \\
(\mathrm{n}=46)\end{array}$ & $\begin{array}{c}\text { Rinoplastia } \\
(\mathrm{n}=17)\end{array}$ & T.ortodôntico \\
\hline
\end{tabular}

$\rho$ de Spearman/P $\rho$ de Spearman/P $\rho$ de Spearman/P $\rho$ de Spearman/P $\rho$ de Spearman/P

\section{Escala Aparência}

\begin{tabular}{|c|c|c|c|c|c|}
\hline Face $\quad(n=97)$ & $0,083 / 0,419$ & $-0,001 / 0,90$ & $-0,001 / 0,990$ & $0,050 / 0,624$ & $-0,435 * * / 0,000$ \\
\hline Nariz $(n=91)$ & $0,016 / 0,878$ & $0,054 / 0,610$ & $-0,192 / 0,088$ & $0,026 / 0,807$ & $-0,168 / 0,127$ \\
\hline Narinas $(\mathrm{n}=92)$ & $-0,002 / 0,987$ & $0,072 / 0,493$ & $-0,114 / 0,280$ & $0,045 / 0,667$ & $-0,303 * * / 0,005$ \\
\hline Dentes $(n=97)$ & $0,004 / 0,971$ & $-0,052 / 0,615$ & $0,042 / 0,682$ & $0,030 / 0,770$ & $-0,362 * * / 0,000$ \\
\hline Lábios (n=95) & $0,011 / 0,919$ & $0,043 / 0,682$ & $0,046 / 0,656$ & $0,028 / 0,787$ & $-0,286 * * / 0,007$ \\
\hline Cicatriz. $(\mathrm{n}=95)$ & $0,051 / 0,625$ & $-0,039 / 0,709$ & $0,033 / 0,750$ & $0,195 / 0,059$ & $-0,167 / 0,119$ \\
\hline Maxilares $(n=96)$ & $0,064 / 0,538$ & $-0,065 / 0,525$ & $0,091 / 0,379$ & $0,077 / 0,454$ & $-0,0225 * / 0,034$ \\
\hline
\end{tabular}

\section{Escala qualidade de vida}

\begin{tabular}{|c|c|c|c|c|c|}
\hline $\begin{array}{l}\text { Dificuldade de fala } \\
(n=95)\end{array}$ & $-0,016 / 0,875$ & $-0,0236 * / 0,021$ & $0,280 * * / 0,006$ & $0,012 / 0,279$ & $-0,348 * * / 0,001$ \\
\hline Psicológico $(\mathrm{n}=100)$ & $0,121 / 0,231$ & $0,056 / 0,581$ & $0,129 / 0,202$ & $0,165 / 0,101$ & $-0,187 / 0,072$ \\
\hline Social $(n=98)$ & $0,113 / 0,270$ & $-0,119 / 0,244$ & $0,255 * / 0,011$ & $-0,087 / 0,395$ & $-0,285 * * / 0,006$ \\
\hline Escolar $(n=998)$ & $0,243 * 0,016$ & $-0,009 / 0,933$ & $0,334 * * / 0,001$ & $0,99 / 0,332$ & $-0.290 * * / 0,005$ \\
\hline
\end{tabular}

\section{Escala de função facial}

Comer/beber $(\mathrm{n}=99) \quad 0,090 / 0,377 \quad-0,072 / 0,479 \quad 0,029 * * / 0,005 \quad-0,047 / 0,647 \quad-0,141 / 0,180$

Fala $(n=97) \quad-0,066 / 0,521 \quad-0,248 * / 0,014 \quad 0,228 * / 0,025 \quad 0,092 / 0,372 \quad-0,306 * * / 0,003$ 
Ainda na Tabela 3, se observa que no domínio Função facial a Escala fala apresentou correlação estatisticamente significante com os tratamentos palatoplastia, enxerto ósseo e tratamento ortodôntico. Na Escala comer/beber foi observada correlação estatisticamente significante apenas com enxerto ósseo.

\section{Discussão}

O autorrelato de pacientes com FLP pode variar de acordo com idade, sexo, tipo de fissura (Riff et al., 2019; Klassen et al., 2018) e o tipo de tratamento (Papi et al., 2015; Eckstein et al., 2011). No presente estudo, a análise dos dados do CLEFT-Q indicou que os tratamentos queiloplastia, palatoplastia e enxerto ósseo causam maior impacto sobre a avaliação da qualidade de vida dos pacientes. Resultados similares foram previamente relatados em outro estudo realizado por Ruiz-Guillén et al. (2021) no qual observaram que todas as dimensões de QV, em pacientes com FLP, apresentaram diferenças estatisticamente significativas, após realização de tratamento cirúrgico e tratamento ortodôntico. Outros estudos corroboram estes achados e indicam o tratamento cirúrgico como modulador da qualidade de vida de pacientes com fissura (Broder et al., 2017; Belluci \& Genaro 2016). Estudos evidenciam que os tratamentos das FLP melhoraram significativamente o bem-estar funcional e emocional, a autoestima e interação social, bem como o funcionamento físico e psicológico e, impactam positivamente na condição de saúde bucal de pacientes (Broder et al., 2017; Belluci \& Genaro, 2016; Eckstein et al., 2011). Os resultados do presente estudo corroboram este dado, uma vez que constatam impacto dos tratamentos em todas as escalas da QV dos pacientes com fissura labiopalatina.

Um achado inusitado foi observado na amostra estudada, onde o tratamento ortodôntico influenciou negativamente todas as Escalas dos três domínios, Aparência, Qualidade de vida e Função facial, afetando principalmente aspectos relacionados à aparência e qualidade de vida. Supomos que seja devido os procedimentos ortodônticos estarem sendo realizados no momento da pesquisa, e que isso possa ter contribuído como uma memória recente de mais fácil acesso para sentidos de vivência, enquanto que a maioria dos demais tratamentos foi realizada em um passado longínquo, na infância. Além disso, presumimos que a idade dos pacientes também possa ter contribuído para este resultado.

No presente estudo os resultados CLEFT-Q mais baixos foram observados no Domínio Aparência, especialmente em relação a nariz e narina. Esta resultado pode ser justificado devido ao elevado percentual da amostra estudada (75\%) composta por crianças e adolescentes de até 17 anos, e se considerarmos que é na infância e início da adolescência, onde a maioria dos indivíduos experimenta estigmas, autopercepção negativa, sendo mais propensos a perceber o processo de tratamento não apenas como insuportavelmente pesado, mas também como causador de seu sentimento de "imperfeição" (Alansari et al. 2014). Na idade adulta, a autopercepção dos participantes melhora, em parte devido à correção cirúrgica definitiva, levando-os a perceber os benefícios do tratamento e a reavaliar o processo de tratamento como satisfatório em vez de penoso.

Sob outra perspectiva, em divergência ao relatado anteriormente, Ward et al. (2013) afirmam que crianças com fissura apresentam menor índice de QV do que o grupo controle, sendo o impacto negativo maior entre os jovens de 15 a 18 anos que nos grupos mais jovens. Desse modo, observa-se que não há consenso em alguns aspectos relacionados à compreensão de como os indivíduos com fissura labiopalatina (FLP) percebem e vivenciam seu processo de tratamento e como essas percepções e experiências mudam ao longo da vida (Alansari et al. 2014).

Vale salientar que o instrumento CLEFT-Q foi considerado por pacientes como sendo o que melhor avalia sua percepção para informar ao profissional de saúde, uma vez que possui informações mais detalhadas e abrangentes nos itens avaliados (Stiernman et al., 2021). Este dado reforça a necessidade de incluir a perspectiva dos pacientes com FLP no processo de avaliação clínica, considerando que há evidências de que tal prática contribui para a melhora da qualidade de vida relacionada à saúde, bem como contribui na avaliação dos protocolos aplicados (Mulder et al., 2019; Stiernman et al 2021). Os 
profissionais que cuidam de indivíduos com fissura labiopalatina estão começando a reconhecer a importância de compreender o efeito do tratamento de fissuras ao longo da vida, conforme as experiências dos pacientes e as necessidades clínicas percebidas (Alansari et al., 2014).

Como limitação do presente estudo, acreditamos que seja a dificuldade de interpretação do questionário por crianças menores de dez anos. No Brasil e em outros países com elevada iniquidade, há crianças com 8 anos que ainda apresentam dificuldade de leitura, precisando de ajuda para responder o índice, o que pode causar constrangimento. Outro fator limitante consiste na existência de poucos estudos publicados que utilizam o CLEFT-Q, o que dificulta a comparação dos dados.

Diante disso, considerando que ainda são escassos os estudos clínicos com o CLEFT-Q, bem como sua contribuição para os protocolos de tratamento do paciente com FLP, ressalta-se a necessidade de realizar novos estudos, a fim de ampliar a comparação de dados e consolidação de resultados, para que a autopercepção do paciente seja incorporada como uma variável e resultado importante na rotina do tratamento.

\section{Conclusão}

Os resultados obtidos no CLEFT-Q sugerem que os pacientes na amostra estudada sentiam insatisfação quanto à aparência facial e a qualidade de vida estava afetada em aspectos relacionados ao estresse de fala e relacionamento social, bem como comprometimento da pronúncia de palavras (fala). Por outro lado, sentiam-se satisfeitos psicologicamente, em atividades escolares e ao alimentar-se. Todos os tratamentos impactaram na percepção do paciente quanto à sua aparência, qualidade de vida relacionada à saúde e função facial. A queiloplastia e enxerto ósseo impactaram positivamente na qualidade de vida dos pacientes estudados.

Sugere-se realizar novos estudos com o instrumento CLEFT-Q para que possa ser analisado com mais detalhes o impacto do tratamento ortodôntico em pacientes com FLP, em diferentes faixas etárias.

\section{Referências}

Alansari, R., Bedos, C. \& Allison, P. (2014). Living with cleft lip and palate: the treatment journey. Cleft Palate Craniofac J, 51(2), 222-9. 10.1597/12-255.

Al-Namankany, A., \& Alhubaishi, A. (2018). Effects of cleft lip and palate on children's psychological health: A systematic review. J Taibah Univ Med Sci., 13(4), 311-318. 10.1016/j.jtumed.2018.04.007.

Beluci, M. L. \& Genaro, K. F. (2016). Qualidade de vida de indivíduos com fissura labiopalatina pré e pós-cirúrgica à correção da deformidade dentofacial. Rev Esc Enferm USP, 50 (2), 217-223. 10.1590/S0080-623420160000200006.

Broder, H.L., Wilson-Genderson, M. \& Sischo L. (2017). Oral health-related quality of life in youth receiving cleft-related surgery: Self-report and proxy ratings. Qual Life Res, 26, 859-867. 10.1007/s11136-016-1420-5.

Eckstein DA, Wu RL, Akinbiyi T, Silver L, Taub PJ. Medindo a qualidade de vida em pacientes com fissura labiopalatina: Medidas de resultados relatados pelo paciente atualmente disponíveis. Plast Reconstr Surg. 2011; 128 (5): 518-526. 10.1097/ PRS.0b013e31822b6a67

Eslami, N., Majidi, M. R., Aliakbarian, M. \& Hasanzadeh, N. (2013). Oral health-related quality of life in children with cleft lip and palate. J Craniofac Surg., 24(4):e340-3. 10.1097/SCS.0b013e31828b743b.

Estrela, C. (2018). Metodologia Científica: Ciência, Ensino, Pesquisa. Editora Artes Médicas.

Klages, U., Claus, N., Wehrbein, H. \& Zentner, A. (2006). Development of a questionnaire for assessment of the psychosocial impact of dental aesthetics in young adults. EUR. J. Orthod., 28(2), 103-111. 10.1093/ejo/cji083.

Klassen, A. F., Riff, K., Longmire, N. M., Albert, A., Allen, G. C., Aydin, M. A., Baker, S. B., Cano, S. J., Chan, A. J., Courtemanche, D. J., Dreise, M. M., Goldstein, J. A., Goodacre, T., Harman, K. E., Munill, M., Mahony, A. O., Aguilera, M. P., Peterson, P., Pusic, A. L., Slator, R., Stiernman, M., Tsangaris, E., Tholpady, S. S., Vargas, F. \& Forrest, C. R. (2018). Psychometric findings and normative values for the CLEFT-Q based on 2434 children and young adult patients with cleft lip and/or palate from 12 countries. CMAJ: Canadian Medical Association journal = journal de l'Association medicale canadienne, 190(15), E455-E462. 10.1503/cmaj.170289.

Kuwada, C., Ariji, Y., Kise, Y., Funakoshi, T., Fukuda, M., Kuwada, T., Gotoh, K., \& Ariji, E. (2021). Detection and classification of unilateral cleft alveolus with and without cleft palate on panoramic radiographs using a deep learning system. Sci Rep., 11(1):16044. 10.1038/s41598-021-95653-9. 
Lewis, C. W., Jacob, L. S., \& Lehmann, C. U. (2017). The Primary Care Pediatrician and the Care of Children With Cleft Lip and/or Cleft Palate. AAP SECTION ON ORAL HEALTH. Pediatrics, 139(5):e20170628. 10.1542/peds.2017-0628.

Ministério da Saúde Brasil. Disponível em: http://www.blog.saude.gov.br/index.php/materias-especiais/51968-materia-especial-no-brasil-nasce-uma-criancacom-fissura-labiopalatinas-a-cada-650-nascimentos. Acessado em 15 de agosto de 2021.

Moi, A. L., Gjengedal, H., Lybak, K. \& Vindenes H. (2020). "I smile, but Without Showing My Teeth": The Lived Experience of Cleft, Lip, and Palate in Adults. Fenda palatina Craniofac J ., 57(7): 799-807. 10.1177 / 1055665620922096.

Mulder, F. J., Mosmuller, D. G. M., de Vet, R. H. C. W. \& Don Griot, J. P. W. (2019). Aesthetics Assessment and Patient Reported Outcome of Nasolabial Aesthetics in 18-Year-Old Patients With Unilateral Cleft Lip. Cleft Palate Craniofac J., 56(8):1058-1064. 10.1177/1055665619832439.

Nordenram, G., Davidson, T., Gynther, G., Helgesson, G., Hultin, M., Jemt, T., Lekholm, U., Nilner, K., Norlund, A., Rohlin, M., Sunnegårdh-Grönberg, K. \&Tranæus S. (2013). Qualitative studies of patients' perceptions of loss of teeth, the edentulous state and prosthetic rehabilitation: a systematic review with meta-synthesis. Acta Odontol. Scand., 71, 937-951. 10.3109/00016357.2012.734421.

Ono, S., Ishimaru, M., Matsui, H., Fushimi, K. \& Yasunaga, H. Effect of Hospital Volume on Outcomes of Surgery for Cleft Lip and Palate.(2015). J. Oral Maxillofac. Surg., 73 : 2219-2224. 10.1016/ j.joms.2015.04.003.

Papi, P., Giardino, R., Sassano, P., Amodeo, G., Pompa, G. \& Cascone, P. (2015). Oral health related quality of life in cleft lip and palate patients rehabilitated with conventional prostheses or dental implants. J Int Soc Prev Community Dent.,5(6):482-7. 10.4103/2231-0762.168645.

Raghavan, U., Vijayadev, V., Rao, D. \& Ullas, G. (2018). Postoperative Management of Cleft Lip and Palate Surgery. Facial Plast Surg, 34(6):605-611. $10.1055 / \mathrm{s}-0038-1676381$.

Rando, G. M., Jorge, P. K., Vitor, L. L. R., Carrara, C. F. C., Soares, S., Silva, T. C., Rios, D., Machado, M. A. A. M., Gavião, M. B. \& Oliveira, T. M. (2018). Oral health-related quality of life of children with oral clefts and their families. J Appl Oral Sci., 1;26:e20170106. 10.1590/1678-7757-2017-0106.

Ruiz-Guillén, A., Suso-Ribera, C., Romero-Maroto, M., Gallardo, C. \& Peñacoba, C. (2021). Perception of quality of life by children and adolescents with cleft lip/palate after orthodontic and surgical treatment: gender and age analysis. Prog Orthod,22(1):10. 10.1186/s40510-021-00354-8.

Sato, Y., Yoshioka, E., Saijo, Y., Miyamoto, T., Sengoku, K., Azuma, H., Tanahashi, Y., Ito, Y., Kobayashi, S., Minatoya, M., Bamai, Y. A., Yamazaki, K., Itoh, S., Miyashita, C., Araki, A. \& Kishi, R. Japan Environment and Children's Study (JECS) Group. (2021). Population Attributable Fractions of Modifiable Risk Factors for Nonsyndromic Orofacial Clefts: A Prospective Cohort Study From the Japan Environment and Children's Study. J Epidemiol., 31(4):272-279. 10.2188/jea.JE20190347.

Sischo, L., Wilson-Genderson, M. \& Broder, H. L. Quality-of-Life in Children with Orofacial Clefts and Caregiver Well-being. Journal of Dental Research. 2017; 96:1474 -1481. 10.1177/0022034517725707.

Stiernman, M., Klintö, K., Persson, M. \& Becker, M. (2021). Comparison of Corresponding Scores From the Cleft Hearing Appearance and Speech Questionnaire (CHASQ) and CLEFT-Q in Swedish Patients With Cleft Lip and/or Palate. Cleft Palate Craniofac J., 58(6):736-745. $10.1177 / 1055665620964124$.

Tsangaris, E., Riff, K. W. Y. W., Vargas, F., Aguilera, M. P., Alarcón, M. M., Cazalla, A. A., Thabane, L., Thoma, A. \& Klassen, A. F. (2017). Translation and cultural adaptation of the CLEFT-Q for use in Colombia, Chile, and Spain. Health Qual Life Outcomes.,15(1):228. 10.1186/s12955-017-0805-7.

Ward, J. A., Vig, K. W. L., Firestone, A. R., Mercado, A., Da Fonseca, M. \& Johnston, W. (2013). Oral health-related quality of life in children with orofacial clefts.Cleft Palate Craniofacial J., 50 (2): 174-181. 10.1597 / 11-055.

Riff, K. W. Y., Tsangaris, E., Forrest, C. R., Goodacre, T., Longmire, N. M., Allen, G., Courtemanche, D. J., Goldstein, J., O'Mahony, A., Pusic, A. L., Slator, R., Swan, M. C., Thoma, A., Vargas, F. \& Klassen, A. F. (2019). CLEFT-Q: Detecting Differences in Outcomes among 2434 Patients with Varying Cleft Types. Plast Reconstr Surg., 144(1):78e-88e. 10.1097/PRS.0000000000005723. 